\title{
Moving to a Personalized Approach in Respiratory Medicine. From Academic Research to Regulatory Intervention
}

\author{
Mario Cazzola * \\ Department of Experimental Medicine, University of Rome Tor Vergata, Roma, Italy
}

Keywords: chronic obstructive pulmonary disease (COPD), asthma, lung disease, biotechnolgy, idiopathic pulmonary fibrosis (IPF), interstitial lung disease (ILD), pulmonary hyperetnsion

In recent years, interest in respiratory diseases has increased significantly after a long period of relative stagnation that followed the apparent control of tuberculosis. Indeed, for decades the focus of researchers was mainly on bronchial asthma, with little interest in COPD, and minimal attention on other lung diseases. Despite the widespread prevalence of pulmonary diseases, there has been, and still is, a considerable delay in understanding their etiopathogenesis, and, as a result, the therapeutic approach to these diseases has not developed adequately. For as long as anyone can remember, it has generally been limited to the use of bronchodilators, corticosteroids, mucolytics and antibiotics.

Recent advances in biomedical research and bioinformatics approaches, in particular the commodification of high-throughput biotechnology, are now enabling the understanding of various aspects of biological systems. Insights generated by these advances have gone beyond the limitations of simplistic hypotheses. The obvious consequence of this is that the fundamental mechanisms that regulate biological processes in the lungs are increasingly being discovered, and, as a cascade, several disease endotypes and new therapies that may be effective in selected individuals are also being identified (Hemnes, 2018). Nonetheless, these approaches still have serious limitations due to a high chance of potentially false discovery with low patient numbers, inexact phenotyping, and bioinformatics challenges.

However, the broadening of our knowledge of the underlying mechanisms involved in at least some

Edited and reviewed by: Gianluca Trifirò,

University of Verona, Italy

*Correspondence: Mario Cazzola mario.cazzola@uniroma2.it

Specialty section: This article was submitted to Respiratory Drug Safety,

a section of the journal Frontiers in Drug Safety and Regulation

Received: 03 August 2021 Accepted: 07 September 2021

Published: 29 October 2021

Citation:

Cazzola M (2021) Moving to a Personalized Approach in Respiratory Medicine. From Academic Research to

Regulatory Intervention.

Front. Drug. Saf. Regul. 1:752581. doi: $10.3389 /$ fdsfr.2021.752581 phenotypes and endotypes of asthma and/or COPD has provided access to innovative therapies, especially for asthma (Cazzola et al., 2017; Martin et al., 2019; Rogliani et al., 2020; Cazzola et al., 2021; Matera et al., 2021). Unfortunately, however, it is becoming increasingly accepted that asthma and COPD are chronic inflammatory airway disorders that are highly heterogeneous in terms of pathogenic manifestations, symptom severity and outcome (Sterk, 2016). There is growing evidence that asthma and COPD are umbrella terms used to group endotypes characterised by different underlying mechanisms (Rogliani et al., 2016). These endotypes probably represent diseases that are different and therefore each characterised by clinical and pathological heterogeneity, although there may also be overlapping features (Rogliani et al., 2016).

Idiopathic pulmonary fibrosis (IPF), a chronic interstitial lung disease (ILD) with a poor prognosis, which has received much attention in recent years, is also difficult to classify because it is extremely heterogeneous in terms of clinical progression rates, worsening in lung function decline, and treatment response (Clarke et al., 2017). IPF represents the archetype of progressive fibrosing ILD, but many patients with other ILD subtypes also develop progressive fibrosing ILD (Bowman et al., 2021). Furthermore, the variability in clinical progression with rapid deterioration and death within a few months in some patients, while in other patients there is a slower decline in disease progression and relatively limited associated disability, or even periods of relative stability alternating with periods of acute respiratory decline, suggests that it is likely that the biological factors or drivers involved may be different (Clarke et al., 2017). In any case, predicting a progressive phenotype remains difficult. 
Pulmonary hypertension $(\mathrm{PH})$ is also a heterogeneous disorder in which overlapping syndromes with different origins may be present simultaneously (Barnes and Dweik, 2014). This implies that the pathobiology is equally heterogeneous and, consequently, $\mathrm{PH}$ presents with many phenotypes of which some have already been identified, while others have only been proposed (Dweik et al., 2014). It is likely that the vascular remodelling that characterises chronic $\mathrm{PH}$ may be the result of dynamic interactions over time between specific cell types involved and their surrounding matrix and inflammatory microenvironment (Stenmark et al., 2018). Using single cell genomics, it is possible to precisely map cellular heterogeneity, the contribution of different lung cell types and the underlying molecular signatures that condition the vascular remodelling process in PH (Morrell et al., 2019). An exciting finding is the identification of pharmacologically modulable signalling hubs, transcriptional regulators and epigenetic enzymes that are specifically dysregulated in $\mathrm{PH}$ vascular cells. It is potentially useful for designing therapeutic approaches targeting specific disease subpopulations in $\mathrm{PH}(\mathrm{Li}$ et al., 2020). Nevertheless, at present time, PH remains a progressive, fatal disease with a fair amount of heterogeneity in response to pharmacotherapy. Therefore, even for $\mathrm{PH}$ there is a clear need to define clinical and, in this case, hemodynamic subphenotypes.

These issues explain why respiratory drug development is difficult, but the lack of available treatments can perhaps be partly attributed to a shortage of critical tools in the areas of biomarkers and clinical outcome measures.

The identification of prognostic disorder/disease progressionspecific biomarkers that predict disease course, the creation of scoring and risk stratification systems and the development of carefully controlled studies that do not exclude disease subtypes are now an absolute need (Wheelock et al., 2013; Cazzola et al., 2019; Bowman et al., 2021). These biomarkers could also help in defining populations that will derive most benefit from a drug, guiding the clinical care and monitoring adverse events in individuals with such disorders/diseases. Moreover, biomarkers can reflect disease course. Carefully selected biomarkers are helpful in the early stages of clinical trials, such as proof-ofaction or proof-of-concept studies, to drug development as they allow a clearer definition of the target population that has the highest potential for benefit and the lowest risk of developing unwanted adverse reactions (Barnes et al., 2006; Gromova et al., 2020). This helps to make a rational decision on further drug development. Biomarkers can also be used at different stages of clinical trials to understand the mechanism of action of a drug or to confirm its supposed mode of action (Barnes et al., 2006; Gromova et al., 2020). In addition, phase 3 studies conducted for the development of new therapies may be useful to evaluate in parallel, but independently, a new biomarker proposed as an exploratory endpoint (Barnes et al., 2006). This approach increases the power and robustness of the validation and, if the result is significant, the biomarker can be elevated to surrogate endpoint status (Cordis Eu research results, 2020). However, developing a biomarker is intellectually complicated, operationally demanding and usually very expensive, with a real risk of failure.
In any case, the use of biomarkers is useful for determining the benefit-risk profile of a drug in development, allowing precise patient stratification and reduced number of patients needed to show clinical benefit and non-inferiority in clinical trials. It therefore allows easier decision-making by regulatory agencies (Gromova et al., 2020).

The big problem in respiratory medicine is that there are too few validated biomarkers. In asthma, several biomarkers are available such that a type 2 biomarker strategy, with FeNO, eosinophils in blood and periostin in serum, has already been proposed to determine when to use an ICS or increase its dose (Cazzola et al., 2021). In contrast, in COPD, the European Medicines Agency and the US Food and Drug Administration believe that fibrinogen is still the only circulating biomarker to be used to identify patients for inclusion in clinical trials, even though conflicting data from several studies negate its robustness as a COPD biomarker, suggesting that there is an urgent need to identify and validate new circulating biomarkers that may be of real value (Cazzola et al., 2019). However, even in asthma, defining the presence of eosinophilic airway inflammation and predicting response to corticosteroid therapy requires further investigation to accurately determine FeNO cut-off points and blood eosinophil counts (Cazzola et al., 2021). Furthermore, it is imperative to prospectively evaluate the biomarker-guided approach on a wider range of patients to better understand the efficacy and safety of this approach (Cazzola et al., 2021).

Several molecules have shown potential value as biomarkers in IPF, but their verified accuracy in Asian populations has not yet been confirmed in Caucasian cohorts for routine application in the management of IPF (Stainer et al., 2021). Furthermore, none of these biomarkers have been validated in large clinical trials. An index composed of a set of separately studied biomarkers could improve the diagnostic accuracy in distinguishing IPF from other ILDs or healthy controls but, so far, has shown controversial results (Guiot et al., 2017). Thus, even for this type of diseases the identification of valid biomarkers remains an unmet need.

With regard to clinical outcome measures, despite the great efforts made over the years, the choice of the primary efficacy endpoint is one of the most critical steps. This is particularly true regarding ILDs, for which we still consider decline in forced vital capacity (FVC) as an appropriate endpoint in IPF drug development, with an absolute decline in FVC of more than $10 \%$ significantly associated with an increase in the risk of 1-year mortality (du Bois et al., 2011), whereas a decline of 5-10\% would not be significantly associated with death (Fernández Fabrellas et al., 2018). However, in patients with mild to moderate IPF, clinical deterioration and physiological changes over time are minimal (Spagnolo et al., 2019). Therefore, the nature of the disease makes it difficult to detect large changes in lung function using pulmonary function tests even when they are grouped into a composite physiological index based for example on the predicted \% diffusing capacity of the lung for carbon monoxide $\left(\mathrm{DL}_{\mathrm{CO}}\right), \mathrm{FVC}$ and forced expiratory volume in $1 \mathrm{~s}$ $\left(\mathrm{FEV}_{1}\right)$ (Spagnolo et al., 2019). It is indeed problematic to identify robust predictors of IPF worsening both at baseline and over the course of the disease regardless of the various endpoint 
combinations used (Spagnolo et al., 2019). There is, therefore, an urgent and essential need to develop measures that at least better assess meaningful outcomes for patients (including effects on symptoms and quality of life).

In asthma and COPD, there is more evidence about the clinical outcome measures to be used, with constructive input from different regulatory authorities (Haarst et al., 2019; Collacott et al., 2021). However, there is still a need for a standardized approach to the assessment of asthma/COPD symptoms and their impact on heath related quality of life (HRQoL) from the patient's perspective. Indeed, we do not yet have validated patient-reported outcomes (PROs) available for inclusion in clinical trials that assess the impact of treatment but also the role of key patient aspects such as sleep, fatigue, and activities of daily living (Afroz et al., 2020).

The real problem, however, lies in the fact that the outcomes usually used in trials involving patients with asthma or COPD and approved by the regulatory authorities consider these patients as all affected by asthma or COPD as single, distinct diseases, whereas as it has already been pointed out, the umbrella terms asthma and COPD cover various clinical entities with multiple causes resulting in airflow limitation (Rogliani et al., 2016). These different clinical entities are referred to as endotypes, i.e., disease subtypes defined functionally and pathologically by a molecular mechanism or response to treatment (Cazzola et al., 2017; Cazzola et al., 2021). Endotypes should be identified by means of validated genetic or blood biomarkers, but as pointed out above, we are still a long way from having credible and above all validated biomarkers available.

With the current state of knowledge, future treatments could be classified into two fields of research depending on how asthma, COPD, and ILD are defined, and the types of therapies used.

The existing approach with approved therapies indicates that we are considering and treating these disorders as single endotypes. However, the treatments currently used certainly improve long-term symptoms, and even survival, but are unable to significantly interfere with the variability of the various characteristics of these disorders that differ between patients but also over time in the same patient (Cazzola et al., 2021). Since modern medicine tends to offer the patient a proactive and personalised approach that starts with a precision diagnosis and tends towards personalised treatment, this inability of existing therapies is certainly a major limitation (Cazzola et al., 2021).

An alternative approach sought to understand the heterogeneity of the disorder/disease with the aim of using biomarkers and clinical parameters to stratify it into several endotypes (Clarke et al., 2017). It is clearly necessary to go

\section{REFERENCES}

Afroz, N., Gutzwiller, F. S., Mackay, A. J., Naujoks, C., Patalano, F., and Kostikas, K. (2020). Patient-Reported Outcomes (PROs) in COPD Clinical Trials: Trends and Gaps. Copd 15, 1789-1800. doi:10.2147/ copd.s235845 beyond clinical features and physiological and inflammatory data, which are no longer sufficient (Cazzola et al., 2021). There is a need for identifying the mechanisms that drive the pathologic process in each endotype of asthma, COPD and ILD. When this is possible, a personalized medicine for treatment of these disorders/diseases will surely be developed. This will not only enable more precise treatment prescriptions, but also identify new traits/targets, which is likely to mean the development of new endotype-specific therapies (Cazzola et al., 2021). Obviously, only specifically designed clinical studies will be able to clarify whether and how well this approach works.

The endotype approach is increasingly expanding with a better understanding of the complexity and granularity of molecular data thanks to real recent technological advances. These have enabled the development of "multi-omic" platforms that allow a large set of analytes, typically of a specific type, to be measured on a single sample (Cazzola et al., 2021). It should be noted, however, that although the role of "-omics" in the field of respiratory medicine research is increasingly accepted, various methodological, practical and ethical challenges still preclude the proper analysis, interpretation and clinical application of “-omics" data (Cazzola et al., 2021).

Personalized medicine offers a new paradigm for the development of safer and more effective drugs for specific populations and for a better practice of medicine. However, there are some obstacles to its application that need to be removed. Indeed, regulatory requirements are uncertain and, in any case, legal protections to prevent genetic discrimination are incomplete (Aroh and Cata, 2017). Moreover, apart from the lack of a comprehensive health information technology system, insurance reimbursement for diagnostic tests related to preventive care is totally insufficient (Aroh and Cata, 2017). Therefore, there is a need for decisive action at both the regulatory and academic levels. Regulatory authorities should lead the drafting of official documents standardizing the development of "-omics" techniques in respiratory medicine, obviously with the involvement of the main scientific societies. The academic body should develop a new medical education system that teaches doctors how to implement their daily practice by considering the diagnostic procedures of personalised medicine or the impact of pharmacogenomics (Aroh and Cata, 2017).

\section{AUTHOR CONTRIBUTIONS}

The author confirms being the sole contributor of this work and has approved it for publication.

Aroh, A., and Cata, T. (2017). Personalized Medicine and Stakeholders' Perceptions. Am. J. Manage. 17 (1), 10-18. https://articlegateway.com/index. php/AJM/article/view/1761f

Barnes, J., and Dweik, R. A. (2014). Is Pulmonary Hypertension a Metabolic Disease?. Am. J. Respir. Crit. Care Med. 190 (9), 973-975. doi:10.1164/rccm.201409-1702ed Barnes, P. J., Chowdhury, B., Kharitonov, S. A., Magnussen, H., Page, C. P., Postma, D., et al. (2006). Pulmonary Biomarkers in Chronic Obstructive Pulmonary 
Disease. Am. J. Respir. Crit. Care Med. 174 (1), 6-14. doi:10.1164/rccm.200510$1659 \mathrm{pp}$

Bowman, W. S., Echt, G. A., and Oldham, J. M. (2021). Biomarkers in Progressive Fibrosing Interstitial Lung Disease: Optimizing Diagnosis, Prognosis, and Treatment Response. Front. Med. (Lausanne) 8, 680997. doi:10.3389/ fmed.2021.680997

Cazzola, M., Calzetta, L., Rogliani, P., and Matera, M. G. (2017). The Challenges of Precision Medicine in COPD. Mol. Diagn. Ther. 21 (4), 345-355. doi:10.1007/ s40291-017-0266-z

Cazzola, M., Ora, J., Cavalli, F., Rogliani, P., and Matera, M. G. (2021). Treatable Mechanisms in Asthma. Mol. Diagn. Ther. 25 (2), 111-121. doi:10.1007/ s40291-021-00514-w

Cazzola, M., Puxeddu, E., Ora, J., and Rogliani, P. (2019). Evolving Concepts in Chronic Obstructive Pulmonary Disease Blood-Based Biomarkers. Mol. Diagn. Ther. 23 (5), 603-614. doi:10.1007/s40291-019-00413-1

Clarke, D. L., Murray, L. A., Crestani, B., and Sleeman, M. A. (2017). Is Personalised Medicine the Key to Heterogeneity in Idiopathic Pulmonary Fibrosis?. Pharmacol. Ther. 169, 35-46. doi:10.1016/j.pharmthera.2016.09.010

Collacott, H., Zhang, D., Heidenreich, S., and Tervonen, T. (2021). A Systematic and Critical Review of Discrete Choice Experiments in Asthma and Chronic Obstructive Pulmonary Disease. Patient. doi:10.1007/s40271-021-00536-w

Cordis Eu research results Development of Sensitive and Validated Clinical Endpoints in Primary Sjögren's Syndrome (pSS). Available at: https://cordis. europa.eu/programme/id/H2020_IMI2-2017-12-03 (Accessed July 30, 2021).

du Bois, R. M., Weycker, D., Albera, C., Bradford, W. Z., Costabel, U., Kartashov, A., et al. (2011). Ascertainment of Individual Risk of Mortality for Patients with Idiopathic Pulmonary Fibrosis. Am. J. Respir. Crit. Care Med. 184 (4), 459-466. doi:10.1164/rccm.201011-1790oc

Dweik, R. A., Rounds, S., Erzurum, S. C., Archer, S., Fagan, K., Hassoun, P. M., et al. (2014). An Official American Thoracic Society Statement: Pulmonary Hypertension Phenotypes. Am. J. Respir. Crit. Care Med. 189 (3), 345-355. doi:10.1164/rccm.201311-1954st

Fernández Fabrellas, E., Peris Sánchez, R., Sabater Abad, C., and Juan Samper, G. (2018). Prognosis and Follow-Up of Idiopathic Pulmonary Fibrosis. Med. Sci. 6 (2), 51. doi:10.3390/medsci6020051

Gromova, M., Vaggelas, A., Dallmann, G., and Seimetz, D. (2020). Biomarkers: Opportunities and Challenges for Drug Development in the Current Regulatory Landscape. Biomark Insights 15, 1177271920974652. doi:10.1177/ 1177271920974652

Guiot, J., Moermans, C., Henket, M., Corhay, J.-L., and Louis, R. (2017). Blood Biomarkers in Idiopathic Pulmonary Fibrosis. Lung 195 (3), 273-280. doi:10.1007/s00408-017-9993-5

Haarst, A., McGarvey, L., and Paglialunga, S. (2019). Review of Drug Development Guidance to Treat Chronic Obstructive Pulmonary Disease: US and EU Perspectives. Clin. Pharmacol. Ther. 106 (6), 1222-1235. doi:10.1002/cpt.1540

Hemnes, A. R. (2018). Using Omics to Understand and Treat Pulmonary Vascular Disease. Front. Med. 5, 157. doi:10.3389/fmed.2018.00157

Li, Q., Meng, L., and Liu, D. (2020). Screening and Identification of Therapeutic Targets for Pulmonary Arterial Hypertension through Microarray Technology. Front. Genet. 11, 782. doi:10.3389/fgene.2020.00782
Martin, R. J., Bel, E. H., Pavord, I. D., Price, D., and Reddel, H. K. (2019). Defining Severe Obstructive Lung Disease in the Biologic Era: an Endotype-Based Approach. Eur. Respir. J. 54 (5), 1900108. doi:10.1183/13993003.00108-2019

Matera, M. G., Calzetta, L., Annibale, R., Russo, F., and Cazzola, M. (2021). Classes of Drugs that Target the Cellular Components of Inflammation under Clinical Development for COPD. Expert Rev. Clin. Pharmacol. 14, 1015-1027. doi:10.1080/17512433.2021.1925537

Morrell, N. W., Aldred, M. A., Chung, W. K., Elliott, C. G., Nichols, W. C., Soubrier, F., et al. (2019). Genetics and Genomics of Pulmonary Arterial Hypertension. Eur. Respir. J. 53 (1), 1801899. doi:10.1183/13993003.018992018

Rogliani, P., Calzetta, L., Matera, M. G., Laitano, R., Ritondo, B. L., Hanania, N. A., et al. (2020). Severe Asthma and Biological Therapy: when, Which, and for Whom. Pulm. Ther. 6 (1), 47-66. doi:10.1007/s41030-019-00109-1

Rogliani, P., Ora, J., Puxeddu, E., and Cazzola, M. (2016). Airflow Obstruction: Is it Asthma or Is it COPD?. Copd 11, 3007-3013. doi:10.2147/copd.s54927

Spagnolo, P., Cocconcelli, E., and Cottin, V. (2019). "Clinical Trials in IPF: What Are the Best Endpoints?," in Idiopathic Pulmonary Fibrosis. A Comprehensive Clinical Guide. Editors K. Meyer and S. D. Nathan. 2nd edn. (Switzerland: Springer Nature), 433-453. doi:10.1007/978-3-319-99975-3_19

Stainer, A., Faverio, P., Busnelli, S., Catalano, M., Della Zoppa, M., Marruchella, A., et al. (2021). Molecular Biomarkers in Idiopathic Pulmonary Fibrosis: State of the Art and Future Directions. Ijms 22 (12), 6255. doi:10.3390/ijms22126255 Stenmark, K. R., Frid, M. G., Graham, B. B., and Tuder, R. M. (2018). Dynamic and Diverse Changes in the Functional Properties of Vascular Smooth Muscle Cells in Pulmonary Hypertension. Cardiovasc. Res. 114 (4), 551-564. doi:10.1093/ $\mathrm{cvr} / \mathrm{cvy} 004$

Sterk, P. J. (2016). Chronic Diseases like Asthma and COPD: Do They Truly Exist?. Eur. Respir. J. 47 (2), 359-361. doi:10.1183/13993003.01930-2015

Wheelock, C. E., Goss, V. M., Balgoma, D., Nicholas, B., Brandsma, J., Skipp, P. J., et al. (2013). Application of 'omics Technologies to Biomarker Discovery in Inflammatory Lung Diseases. Eur. Respir. J. 42 (3), 802-825. doi:10.1183/ 09031936.00078812

Conflict of Interest: The author declares that the research was conducted in the absence of any commercial or financial relationships that could be construed as a potential conflict of interest.

Publisher's Note: All claims expressed in this article are solely those of the authors and do not necessarily represent those of their affiliated organizations, or those of the publisher, the editors, and the reviewers. Any product that may be evaluated in this article, or claim that may be made by its manufacturer, is not guaranteed or endorsed by the publisher.

Copyright $(2021$ Cazzola. This is an open-access article distributed under the terms of the Creative Commons Attribution License (CC BY). The use, distribution or reproduction in other forums is permitted, provided the original author(s) and the copyright owner(s) are credited and that the original publication in this journal is cited, in accordance with accepted academic practice. No use, distribution or reproduction is permitted which does not comply with these terms. 